\title{
Tracer particle momentum effects in vortex flows
}

\author{
DAVID M. BIRCH AND NICHOLAS MARTIN \\ Department of Mechanical Engineering Sciences, \\ University of Surrey, Guildford, Surrey GU2 7XH UK
}

(Received 31 August 2012, accepted in revised form 4 February 2013)

The measurement of vortex flows with particle-image velocimetry (PIV) is particularly susceptible to error arising from the finite mass of the tracer particles, owing to the high velocities and accelerations typically experienced. A classical model of Stokes-flow particle transport is adopted, and an approximate solution for the case of particle transport within an axisymmetric, quasi-two-dimensional Batchelor $q$-vortex is presented. A generalized expression for the maximum particle tracking error is proposed for each of the velocity components, and the importance of finite particle size distributions is discussed. The results indicate that the tangential velocity component is significantly less sensitive to tracking error than the radial component, and that the conventional particle selection criterion (based on the particle Stokes number) may result in either over- or under-sized particles for a specified allowable error bound. Results were demonstrated by means of PIV measurements carried out in air and water using particles with very different properties.

\section{Introduction}

Vortex flows are of considerable importance not only fundamentally but in industrial and aeronautical applications, owing to the significant role which they can play in mixing and momentum exchange. Wing-tip vortices have historically been of particular interest; these vortices contribute directly toward the drag experienced by an aircraft, and, because of their persistence, impose minimum wake hazard separation distances during landing and take-off operations. These vortex flows have therefore been the subject of a large number of experimental and numerical studies (see, for example, the reviews of Widnall (1975) and Spalart (1998a), and references contained therein).

Computational modelling of vortex flows (such as the direct numerical simulations of Goto (2008) or the large-eddy models of Uzun et al. (2006)) has produced some valuable insight. However, computational resources still limit the Reynolds number of DNS solutions, while large-eddy models will usually require some validation owing to the inherent sensitivity of vortices to small perturbations in the boundary conditions (Bailey et al. 2006). Consequently, wind-tunnel experiments continue to be of significant importance in modelling and understanding these flows.

One of the most common approaches to the experimental characterization of vortex flows is through the use of wake scans. Velocity and pressure field measurements in the wake of an aircraft model are a valuable resource in predicting the performance characteristics of the aircraft (Spalart 1998b; Rossow 1999), as well as a means to investigate the more fundamental nature of vortex flows (see, for example, Bandyopadhyay et al. (1991) and Phillips \& Graham (1984)). However, single-point scans of vortex flows (such as the hot-wire and multi-hole pressure probe data of Beninati \& Marshall (2005), Birch et al. (2004), Birch \& Lee (2005), Chow et al. (1997) and Dacles-Mariani et al. (1995), 
to list but a few) will tend to underpredict vortex strength owing to the smoothing effect of vortex 'meandering', or the random, low-frequency modulation in the trajectory of the vortex centre. While a number of schemes have been proposed to correct single-point scans of vortex wakes for the effect of meandering (such as those of Devenport et al. (1996), Bailey \& Tavoularis (2008) and Iungo et al. (2009)), it has also been shown that, with meandering amplitudes which are large but within the range of those observed experimentally, the reconstruction of the vortex velocity field from point measurements becomes impossible (Birch 2012).

Optical measurement techniques, on the other hand, may be used to collect twoor even three-dimensional velocity fields instantaneously within a vortex wake. Timeresolved PIV provides a very powerful means of obtaining velocity statistics within a vortex nonintrusively, and without any contamination of the data by the random meandering commonly observed in wind-tunnel studies (see Ramasamy et al. (2009), Beresh et al. (2010) and van Jaarsveld et al. (2011), for example). With optical measurements, however, some error is necessarily introduced as a result of the momentum of the tracer particles, and this effect is of particular importance in the measurement of highly vortical flows.

It is therefore common practice, in designing experiments, to model the motion of the tracer particles a priori in order to determine the magnitude of the measurement error expected to be introduced as a result of the difference between the measurable particle velocity and the velocity of the fluid itself. This error estimate is especially important in cases where strong circulation or small length-scales is expected, such as those of aircraft wing-tip and rotorcraft blade-tip vortices, as well as in studies of forced swirling flow (Tari et al. 2010; Zhang \& Sarkar 2012). With the aid of these models, a series of well-accepted criteria for the selection of appropriate seed particles have been established based on the nature of the flow being seeded (Leishman 1996; Greenwell 2002; Melling 1997; Dring 1982). However, there are practical issues affecting particle selection as well; though the tracking fidelity of tracer particles will improve with decreasing size, the light reflected by the particles (and therefore the signal-to-noise ratio in the correlation plane) also decreases rapidly. This is especially important in micro-PIV, tomographic PIV and high-speed PIV applications, where the particle illumination is already limited. A less general, flow-specific model for the selection of tracer particles would therefore be of value in optimizing the particle selection process. Furthermore, seed particles are rarely all of uniform diameter, and will typically exhibit a normal distribution of diameters about the mean, while swirling flows tend to be fairly effective at promoting particle agglomeration (Paiva et al. 2010). A proportion of seed particles are likely to exist within the measurement volume which exceed the maximum allowable size. Since PIV measurement techniques also permit the measurement of particle diameters (Martin et al. 1997), a reliable particle transport model should also be useful in extending the range of particle sizes capable of yielding reasonable results.

The purpose of this study, then, is to develop an analytical model of a typical PIV particle within a vortex in order to (a) establish a set of more precise, flow-specific particle selection criteria for the particular case of vortices, and (b) provide a technique for estimating the maximum measurement error arising from the particle tracking error independently for each of the velocity components obtained from an instantaneous optical field measurement in these flows. 


\section{Vortex model}

\subsection{Simplified flow field and tracer particle dynamics}

The flow field considered is a quasi-two-dimensional, viscous, incompressible Batchelor (1964) $q$-vortex, with a tangential velocity profile of the form

$$
\frac{v(r)}{v_{0}}=\left(1+\frac{1}{2 \alpha}\right) \frac{1}{\eta}\left(1-\exp \left(-\alpha \eta^{2}\right)\right),
$$

where $v_{0}$ is the peak tangential velocity, $\eta=r / r_{c}$ is the nondimensional radial coordinate, and $r_{c}$ is the core radius (so that $v\left(r_{c}\right)=v_{0}$ ), and $\alpha=1.25643$ is a constant defined by the scaling parameters. The Batchelor solution admits a relative axial velocity of the form

$$
\frac{w}{w_{0}}=\exp \left(-\alpha \frac{r^{2}}{r_{0}^{2}}\right),
$$

where $w_{0}$ is the peak axial velocity (which may be positive or negative, and may vary with $z$ ) and $r_{0}$ is a radial scaling factor for the axial profile. The solution also admits a bulk axial translation of the flow field; this is analogous to the free-stream velocity for the case of wing tip vortices, and corresponds simply to a transformation to a moving reference frame. Because this bulk translation has no significant effect on the flow fields, it is often omitted from (2.2).

The Batchelor vortex is used as a good approximation of the flow fields produced by a vortex generator or finite lifting wing; see, for example, the data of Phillips (1981), Devenport et al. (1996), Birch et al. (2004) and references cited therein. Though the Batchelor solution assumes a low Reynolds number, the mean tangential velocity profile also collapses well with the Hoffman \& Joubert (1963) mean high Reynolds-number solution (which also assumes that viscosity dominates for $\eta \leq 0.4$ ), and is therefore representative of a very wide range of typical vortex flows.

With $v$ and $w$ explicitly defined, the radial velocity $u$ is constrained by continuity, so that, in polar coordinates,

$$
\frac{\partial u}{\partial r}+\frac{u}{r}+\frac{1}{r} \frac{\partial v}{\partial \theta}+\frac{\partial w}{\partial z}=0,
$$

where $z$ and $\theta$ are the axial and azimuthal coordinates, respectively.

A very small, spherical particle immersed in a Batchelor vortex will experience Stokes flow, providing that the Reynolds number $R e=U d / \nu$ (where $U$ is the velocity of the particle relative to the fluid, $d$ is the diameter of the particle and $\nu$ is the kinematic viscosity) is of $\mathcal{O}(1)$. The coefficient of drag for the particle is then given by $C_{D}=24 / R e$, and so the drag force $F_{D}$ experienced by the particle will be

$$
F_{D}=3 \pi \nu \rho U d,
$$

where $\rho$ is the fluid density and $\nu$ is the kinematic viscosity. Because the force is proportional to velocity, $F_{D}$ may be decomposed into radial and tangential components, which may be evaluated independently with the corresponding components of velocity. If the Stokes drag force is assumed to dominate in the motion of the particle, then the motion of the particle may be described by the linear momentum equation, as

$$
\begin{aligned}
\frac{d v^{\prime}}{d t} & =-\frac{1}{r} u^{\prime} v^{\prime}+C\left(v-v^{\prime}\right) \\
\frac{d u^{\prime}}{d t} & =\frac{1}{r} v^{\prime 2}+C\left(u-u^{\prime}\right),
\end{aligned}
$$


where $u^{\prime}$ and $v^{\prime}$ are the absolute radial and tangential velocities of the particle, respectively, and

$$
C=\frac{18 \nu}{\phi d^{2}},
$$

is an inverse time scale, where $\phi=\rho^{\prime} / \rho$ is the particle density ratio (see Greenwell (2002) and Melling (1997) for a more detailed derivation).

In order to formulate equations (2.5) and (2.6), it was necessary to assume vanishing initial conditions. In general, initial particle relative velocities will have a significant impact on particle trajectories for some finite time. However, after a sufficient time is allowed to elapse, the relative particle history terms will be of $\mathcal{O}\left(d^{2} v_{0} / \nu r_{c}\right)^{1 / 2}$ and will typically be negligible (see Maxey \& Riley 1983, for an elegant demonstration). Indeed, In experimental measurements, great care must be taken to minimize the local effects of mass and momentum injection from the introduction of particle-laden secondary flows (such as atomizer jets, for example). Not only can these secondary flows significantly alter the flow structures being measured, but PIV measurements must also be independent of the effects of any arbitrary initial particle relative velocities.

\subsection{Additional forces and particle dynamics}

When modelling the motion of tracer particles, it is conventional to assume that the effects of particle rotation, buoyancy and shear are negligible relative to the Stokes drag (Leishman 1996; Greenwell 2002; Melling 1997). However, because of the strong radial gradients characteristic of vortex cores, it is important to verify the validity of these assumptions.

The generalized equations of motion for a small, rigid sphere immersed in Stokes flow were derived by Maxey \& Riley (1983). For a particle undergoing rotation about its own axis while translating through the fluid, the relative lift experienced by the particle was shown to be of $\mathcal{O}\left(\Omega d^{2} / \nu\right)$, where $\Omega$ is the angular velocity of the particle. If the particle is spinning as a consequence of local shear, $\Omega$ is approximated by the local velocity gradient; within the core of a Batchelor vortex, $\Omega$ is of $\mathcal{O}\left(v_{0} / r_{c}\right)$. For typical laboratoryscale measurements, then, the influence of particle spin will be negligible relative to the Stokes drag.

The above treatment assumes that the shear itself does not contribute to the lift, though the effect of shear is expected to be more significant. Saffman (1965) solved explicitly for the lift force experienced by an unconstrained sphere in shearing Stokes flow. Applying this solution to the case of a particle in a vortex core (again taking the local velocity gradient as $v_{0} / r_{c}$ ), the ratio of the lift $F_{L}$ to the Stokes drag may be approximated by

$$
\frac{F_{L}}{F_{D}} \approx 2.15 \frac{d}{r_{c}} \sqrt{R e_{c}},
$$

where $F_{D}$ is given by (2.4). For laboratory-scale flows, $R e_{c}$ will not typically exceed $\mathcal{O}\left(10^{3}\right)$, so the relative lift force will be of $\mathcal{O}\left(10 d / r_{c}\right)$ at most. Since well-resolved measurements necessarily require $d \ll r_{c}$, the lift can normally be neglected.

Finally, the net gravitational and buoyancy force acting on the particle $F_{B}$ may be expressed relative to the Stokes drag as

$$
\frac{F_{B}}{F_{D}}=\frac{1}{18}(1-\phi)\left(\frac{v d}{\nu}\right)\left(1-\frac{v^{\prime}}{v}\right)^{-1}\left(\frac{g d}{v^{2}}\right)
$$

(where a positive $F_{B} / F_{D}$ indicates positive buoyancy, and the flow has been assumed incompressible). The term $v d / \nu$ represents a local particle Reynolds number, and is 

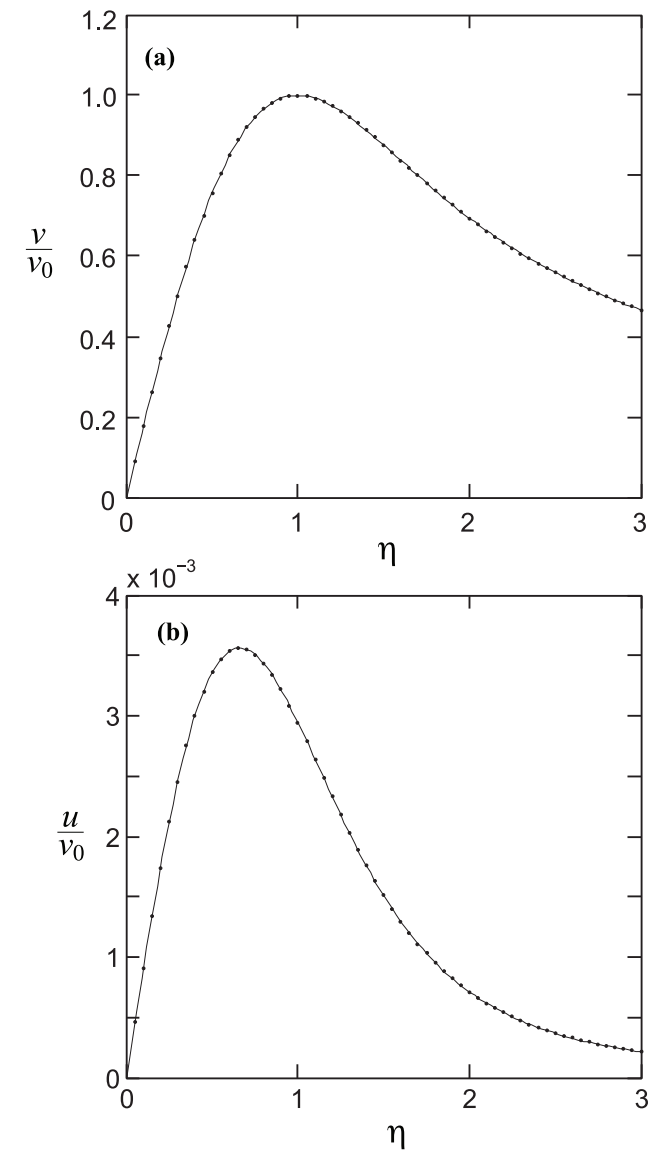

Figure 1. (a) Typical seed particle and fluid velocity as a function of radius, with $u=0$. fluid; •, particle. (b) Radial velocity of particle as a function of radius. from $(3.2) ; \bullet$, numerical solution.

therefore generally expected to be of $\mathcal{O}(1)$. The term $g d / v^{2}$ is a measure of the relative velocity scales, and $\phi g d / v^{2}$ for common PIV particles in laboratory-scale vortex flows will tend to be of $\mathcal{O}\left(10^{-4}\right)$. The force resulting from the net gravitational and buoyancy force may therefore be reasonably neglected if the tangential velocity tracking error is of $\sim \mathcal{O}\left(10^{-3}\right)$ or greater.

\section{Approximate solution}

The tangential velocity from (2.1), together with the radial velocity obtained from (2.2) and (2.3), may be substituted into (2.5) and (2.6), and the system may be solved. For the case of solid-body rotation (so that $u=w=0$ and $v=\zeta r$, where $\zeta$ is a constant), (2.5) and (2.6) may be integrated directly to determine the particle trajectories (see (Kriebel 1961) for this solution); otherwise, the equations may be solved solved numerically.

A typical solution to the system described by (2.5) and (2.6) for the case of an axisymmetric Batchelor $q$-vortex having $u=w=0$ is shown in Figure 1. A vortex with a core Reynolds number $R e_{c}=\Gamma_{c} / \nu$ (where $\Gamma_{c}$ is the circulation around a path of radius $r=r_{c}$ ) of $6 \times 10^{5}$ was modeled, and a value of $C=9.0 \times 10^{4} \mathrm{~s}^{-1}$ was used, corresponding to the case of air seeded with $\sim 2 \mu$ m-diameter oil droplets (similar to those reported by 


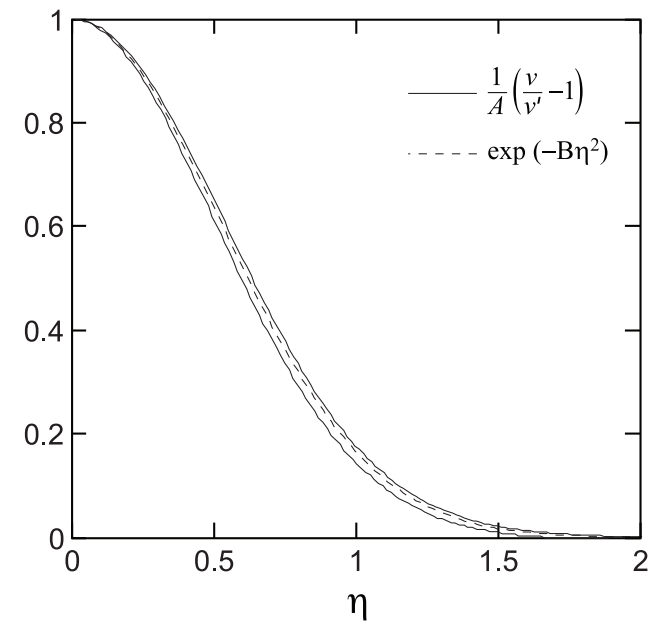

FiguRE 2. Normalized particle tracking error in a typical tangential velocity profile, comparing (3.1) with $B=1.80$ to the numerical solutions for the range $14 \leq C r_{c} / v_{0} \leq 1400$.

Martinelli et al. (2007)). The solution shown was taken at sufficiently large $t$ that the effects of the initial conditions had become negligible. As expected, the particle velocities track the fluid velocity very well in this case, and the tangential velocity profile of the particles is indistinguishable from that of the fluid (Figure $1 \mathrm{a}$ ). As a result of the centrifugal term, the particles experience a very small but nonzero radial velocity, having a maximum of about $0.35 \%$ of $v_{0}$ at $r / r_{c} \approx 0.66$ (Figure $1 \mathrm{~b}$ ).

\subsection{Tangential velocity error}

For the special case when $u=w=0$, the system of equations (2.6) and (2.5) can be shown numerically to admit an approximate solution of the form

$$
\frac{v}{v^{\prime}}-1=A \exp \left(-B \eta^{2}\right)
$$

where $A$ is a parameter dependent upon the flow and particle properties, and represents the maximum error. The form of (3.1) agrees very well with the distribution of tangential velocity tracking error, and the scaling factor $B$ is surprisingly constant over the range of at least $14 \leq C r_{c} / v_{0} \leq 1400$ (broadly representative of the range of $C r_{c} / v_{0}$ encountered in experimental measurements). For all cases tested with $u=w=0, B \sim 1.80$ (Figure $2)$.

One important implication of the good agreement between (3.1) and the numerical solution of the system defined by $(2.1),(2.3),(2.5)$ and (2.6) is that an approximate analytical solution of the system becomes possible. The magnitude of the tracking error $A$ may then be determined from the fluid and particle properties alone, and (3.1) can then provide not only an estimate of the error but could even provide a means of correcting the tangential velocity profile for the effect of particle mass for cases where $A$ is unacceptably large.

To address this problem, $v^{\prime}$ may be isolated from (3.1) and substituted into (2.6). Considering only the case of purely two-dimensional vortex flows (so that $u=0$ ), this yields

$$
\frac{1}{C r_{c} \eta}\left(\frac{v}{1+A \exp \left(-B \eta^{2}\right)}\right)^{2}-u^{\prime}=0,
$$


where the approximation $d u^{\prime} / d t \approx 0$ has been made, as $v^{\prime} \gg u^{\prime}$ for $\eta$ of $\mathcal{O}(1)$, and

$$
\frac{1}{r_{c}} \frac{d u^{\prime}}{d \eta} \ll C
$$

This is equivalent to the assumption that the particles have achieved a 'steady state' in an Eulerian sense, so that their velocity is a function of position alone. Equation (3.2) then provides an explicit expression for $u^{\prime}$ as a function of $v$, and agrees very well with the numerical solution (Figure $1 \mathrm{~b}$ ); note that, for small $A,(3.2)$ reduces to $u^{\prime} \approx v^{2} / C r_{c} \eta$.

Substituting (3.2) into (2.5) provides a single differential equation in $v^{\prime}$ and $v$, as

$$
\begin{aligned}
\frac{d v^{\prime}}{d t}=\frac{u^{\prime}}{r_{c}} \frac{d v^{\prime}}{d \eta}= & C v\left(1-\frac{1}{1+A \exp \left(-B \eta^{2}\right)}\right) \\
& -\frac{1}{C r_{c}^{2} \eta^{2}}\left(\frac{v}{1+A \exp \left(-B \eta^{2}\right)}\right)^{3}
\end{aligned}
$$

The particle tangential velocity $v^{\prime}$ may be expressed as a function of $\eta$ by substituting (2.1) into (3.1), as

$$
v^{\prime}=\left(1+\frac{1}{2 \alpha}\right) \frac{v_{0}}{\eta} \frac{1-\exp \left(-\alpha \eta^{2}\right)}{1+A \exp \left(-B \eta^{2}\right)} .
$$

Differentiating (3.5) with respect to $\eta$, substituting into (3.4) with (3.2) and simplifying,

$$
2 \alpha \exp \left(-\alpha \eta^{2}\right)+2 A B \exp \left(-B \eta^{2}\right) \frac{1-\exp \left(-\alpha \eta^{2}\right)}{1+A \exp \left(-B \eta^{2}\right)}=\frac{A}{A_{0}}
$$

where the substitution

$$
A_{0}=\left(\frac{1+\frac{1}{2 \alpha}}{1+A \exp \left(-B \eta^{2}\right)} \frac{v_{0}}{C r_{c} \eta}\right)^{2} \frac{1-\exp \left(-\alpha \eta^{2}\right)}{\exp \left(-B \eta^{2}\right)}
$$

has been made for convenience.

The error amplitude $A$ may then be obtained from (3.6) as a function of the nondimensional parameters $v_{0} / C r_{c}$ and $\eta$, taking $B=1.80$. It is important to note that though this solution was formulated under the assumption that $A$ is independent of $\eta$, the variation of $A$ with $\eta$ in (3.6) is less than $5 \%$ over the range $0 \leq \eta \lesssim 1.2$ for typical values of $C$ (Figure 3). Because the numerical solution shows the maximum particle tracking error $v / v^{\prime}$ to occur at the centre of the vortex (see Figure 2), a good approximation of the maximum value of $A$ will be obtained as $\eta \rightarrow 0$. Expanding (3.6) about $\eta=0$ and assuming that $A \ll 1$, then (to a second-order approximation),

$$
A=2\left(\alpha+\frac{1}{2}\right)^{2}\left(\frac{v_{0}}{C r_{c}}\right)^{2}
$$

Equation (3.8) demonstrates very close agreement with the value of $A$ satisfying (3.6) for $\eta \rightarrow 0$. Equation (3.8) is also plotted in figure 3 for reference.

Figure 4 shows the variation of $A$ as a function of $v_{0} / C r_{c}$, for values of $A$ as large as $10 \%$. Through this wide range of particle and flow parameters, (3.8) continues to predict $A$ with reasonable accuracy. However, $v_{0}$ and $r_{c}$ cannot be directly obtained from PIV measurements; rather, only the velocities of the particles are available. Equation (3.8) may therefore be further approximated as

$$
A \approx 2\left(\alpha+\frac{1}{2}\right)^{2}\left(\frac{v_{0}^{\prime}}{C r_{c}^{\prime}}\right)^{2}
$$




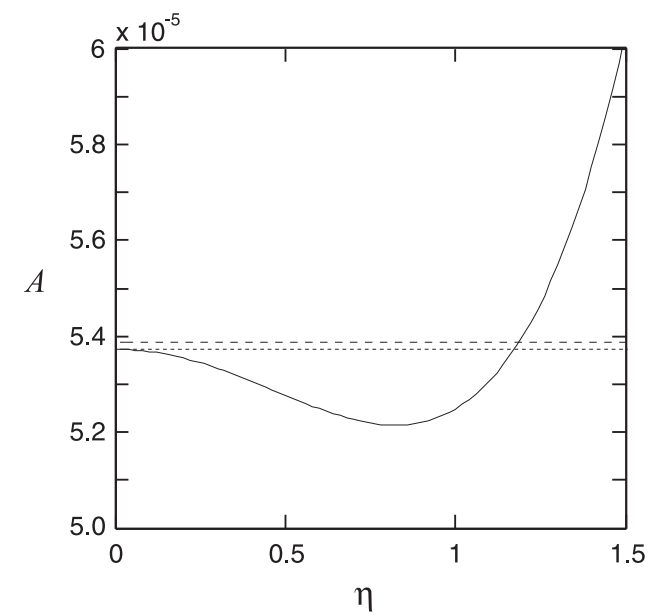

Figure 3. Variation of $A$ with $\eta$ for the case $R e_{c}=6 \times 10^{5}$ and $C r_{c} / v_{0}=340 .-A$ satisfying $(3.6) ;---,(3.8) ;---,\left(v / v^{\prime}-1\right) / \exp \left(-B \eta^{2}\right)$ from the numerical solution to $(2.5)$ and $(2.6)$.

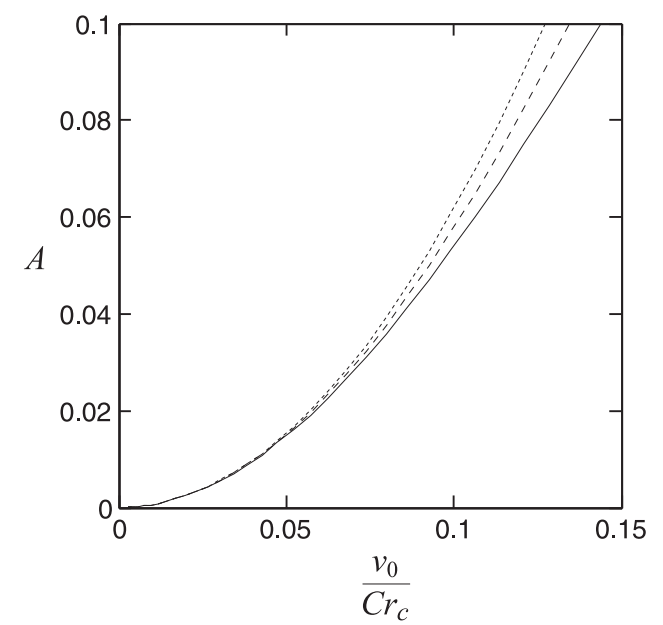

Figure 4. Variation of $A$ with $v_{0} / C r_{c} .-,\left(v / v^{\prime}-1\right) / \exp \left(-B \eta^{2}\right)$ from the numerical solution to $(2.5)$ and $(2.6) ;---,(3.8) ;---,(3.9)$.

where $v_{0}^{\prime}$ is the peak tangential velocity of the particles, and $r_{c}^{\prime}$ is the radius at which this peak velocity is observed. Even with this additional approximation, for a range of $C r_{c} / v_{0}$ of interest to experimentalists, (3.9) is able to predict the peak particle tracking error to within $\sim 5 \%$.

In many cases, the tracking fidelity of a given tracer particle is assessed by means of its Stokes number $S_{k}=\tau^{\prime} / \tau$, where $\tau^{\prime}$ and $\tau$ are the characteristic time-scales of the particle and the fluid, respectively. The characteristic time scale of the particle is generally taken as the characteristic response time of a sphere in Stokes flow to a step-change in flow velocity,

$$
\tau^{\prime}=\frac{4}{C}\left(1-\frac{1}{\phi}\right)
$$

(Tropea et al. 2007). For a Batchelor vortex with $u=w=0$, the local time-scale of the fluid varies with $\eta$, and may be taken as $\tau=r / v$. The Stokes number may then be 
expressed as

$$
S_{k}(\eta)=\frac{4 v_{0}}{C r_{c} \eta^{2}}\left(1-\frac{1}{\phi}\right)\left(1+\frac{1}{2 \alpha}\right)\left(1-\exp \left(-\alpha \eta^{2}\right)\right) .
$$

This expression has a single local maximum at $\eta=0$. Expanding (3.11) about $\eta=0$ to the second order, the maximum Stokes number may be found as

$$
S_{k}(0)=\frac{4 v_{0}}{C r_{c}}\left(1-\frac{1}{\phi}\right)\left(\alpha+\frac{1}{2}\right) .
$$

Combining (3.12) with (3.8), then, demonstrates that for the case of a Batchelor vortex,

$$
S_{k}=2 \sqrt{2 A}\left(1-\frac{1}{\phi}\right)
$$

A maximum value of $S_{k} \lesssim 0.1$ is usually deemed acceptable when a tracking error of less than $\sim 1 \%$ is desired (Tropea et al. 2007). If one assumes that $\phi$ is reasonably large (as it would be for atomized oil droplets in air, for example), (3.13) suggests that a $1 \%$ tracking error in the tangential velocity profile may be achieved for $S_{k}$ as large as 0.28. Since even small increases in particle diameter may greatly improve PIV image quality, this result is significant.

\subsection{Radial velocity error}

For the case of a Batchelor $q$-vortex having $u=0$, any non-zero radial velocities measured using PIV would occur as a result of the particle momentum effects alone. Though vortices with $u=w=0$ are rarely encountered (and have even been shown to be very difficult to produce; see, for example, Phillips \& Graham (1984)), this case is of interest as it provides a means of quantifying the error in the radial velocity component arising from the particle momentum. Interestingly, (3.2) suggests that $u^{\prime}$ will increase as the tangential velocity tracking error decreases.

Because a particle at $\eta=0$ feels no net imbalance of forces, and because the vortex has a vanishing influence upon particles as $\eta \rightarrow \infty, u^{\prime}$ is expected to have a local maximum of $u_{0}^{\prime}$ at some finite positive $\eta_{0}$. This maximum radial velocity may be used as an additional quantification of the error. To obtain an estimate of $u_{0}^{\prime},(3.2)$ may be differentiated with respect to $\eta$. Requiring the resulting expression for $d u^{\prime} / d \eta$ to vanish and assuming that $A \ll 1$ yields,

$$
\frac{4 \alpha}{\exp \left(\alpha \eta_{0}^{2}\right)}-\frac{3}{\eta_{0}^{2}}=0,
$$

which is uniquely satisfied by $\eta_{0}=0.6618$, and is notably independent of $B$. This value of $\eta_{0}$ may then be substituted into (2.1) and (3.2) to obtain an approximation of the maximum radial velocity $u_{0}^{\prime}$ of the particles, as

$$
\frac{u_{0}^{\prime}}{v_{0}}=1.2076 \frac{v_{0}}{C r_{c}}
$$

where the assumption of $A \ll 1$ has again been applied. Comparing (3.15) with (3.12) yields,

$$
S_{k}=5.8 \frac{u_{0}^{\prime}}{v_{0}}
$$

where it has again been assumed that $\phi$ is reasonably large. This result suggests that a maximum tracking error in the radial velocity profile of $1 \%$ of $v_{0}$ would require $S_{k} \leq$ 0.058 . 
It is also very useful to note that (3.2), (3.15) and (2.1) may be combined to yield the approximate normalized radial velocity profile,

$$
\frac{u^{\prime}}{u_{0}^{\prime}}=\frac{1.6183}{\eta^{3}}\left(1-\exp \left(-\alpha \eta^{2}\right)\right)^{2},
$$

where it has been assumed that $A$ is small. The importance of (3.17) is that it is independent of not only $B$, but all experimental parameters as well: since quantities such as $r_{c}$ and $v_{0}$ may be subject to comparatively high experimental uncertainty and $d$ is typically only weakly known, a dimensional approximation of $u^{\prime}(\eta)$ from (3.2) (which is proportional to $d^{2} v_{0}^{2}$ ) will be necessarily poor.

A second estimate of $u^{\prime}$ may also be obtained from (2.5). Differentiating (3.1) with respect to time, an expression for $d v^{\prime} / d t$ may be found as a function of $d v / d t$ and $u$, as

$$
\frac{d v^{\prime}}{d t}=\frac{d v}{d t} \frac{1}{1+A \exp \left(-B \eta^{2}\right)}+\frac{2 A B}{r_{c}} \frac{\eta u^{\prime} v \exp \left(-B \eta^{2}\right)}{\left(1+A \exp \left(-B \eta^{2}\right)\right)^{2}} .
$$

Combining (3.18) with (2.1) and (2.5) allows $u^{\prime}$ to be solved for without further assumptions, as

$$
u^{\prime}=\frac{A C r_{c}}{2 \eta}\left(\alpha \frac{\exp \left(B \eta^{2}\right)}{\exp \left(\alpha \eta^{2}\right)-1}+\frac{A B}{1+A \exp \left(-B \eta^{2}\right)}\right)^{-1} .
$$

Comparing (3.19) to (3.2) then provides one means of assessing how well the momentum equations (2.5) and (2.6) are satisfied by the proposed approximate solution. Figure 5 shows the solutions to (3.2) and (3.19) as functions of $\eta$ for the cases of $C r_{c} / v_{0}=14$ and 1400. The two curves agree reasonably well, despite the high sensitivity of the momentum equations to small changes in the velocity profiles.

\subsection{Implications for continuity}

While three-component and stereoscopic PIV systems are becoming more common, most PIV measurements continue to be collected in two dimensions only. Because vortex flows are generally quasi-two dimensional in nature, there have been efforts to infer $w$ from the $u$ and $v$ fields using the continuity equation. The process of applying continuity to PIV measurements within strong vortical flows has been shown to yield reasonable results (Regunath et al. 2008), and a direct comparison between the inferred and measured outof-plane component has shown very good agreement in certain cases (Brown et al. 2007). Wing-tip vortices, however, tend to exhibit some axial distortion, while small errors in the orientation of the viewing plane can result in significant error when attempting to infer $w$ from continuity within these flows. Nevertheless, techniques for correcting PIV data for finite viewing angles have been demonstrated (see, for example, Grant et al. 1994), so some consideration of the influence of particle momentum upon the inferred axial velocity is warranted.

From (2.3), it is clear that nonzero $u$ necessarily requires nonzero $w$ (if no singularities are admitted); the interrelationship between $u$ and $w$ in vortex development has been reviewed in detail by Phillips \& Graham (1984). Applying the continuity equation to the nonzero $u$ profile arising in PIV measurements entirely as a consequence of the particle mass (Figure 1 b) will therefore result in an 'apparent' or 'false' axial velocity distribution $w^{*}(\eta) \neq 0$ in a Batchelor vortex having $u=w=0$ given by

$$
w^{*}=-\int \frac{1}{r_{c}}\left(\frac{\partial u^{\prime}}{\partial \eta}+\frac{u^{\prime}}{\eta}\right) d z .
$$

as the assumption of axisymmetry requires $\partial / \partial \theta=0$. It should be noted here that the 

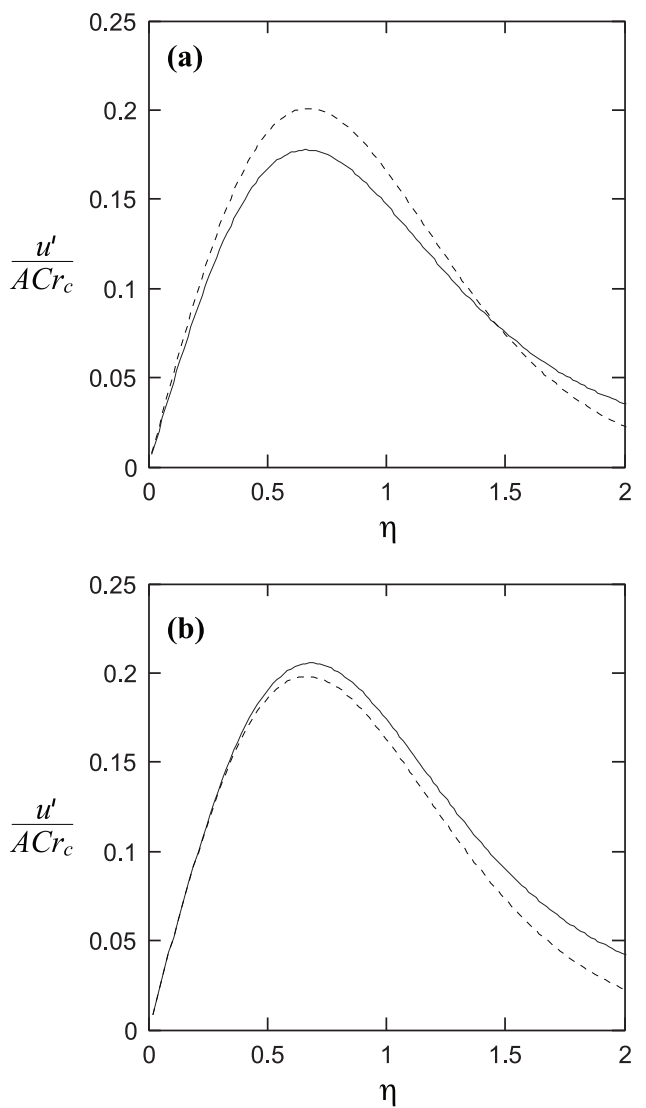

Figure 5. Comparison of $u^{\prime}$ approximations. (a), $C r_{c} / v_{0}=14 ;$ (b), $C r_{c} / v_{0}=1400 .-$, $(3.2) ;---,(3.19)$.

quantity $z$ represents a measure of the degree of development of the 'vortex' described by the particle velocities.

Equation (3.20) can be integrated numerically, and the result is shown in Figure 6 for the case of a typical vortex having $u=0$ so $w(\eta)=w_{0}$. Disturbingly, the distribution agrees very well with $(2.2)$ over the range $0 \leq \eta \lesssim 0.8$ (with $r_{0} / r_{c} \sim 0.71$ ). Over the range $\eta \gtrsim 0.8$, the inferred axial velocity profile exhibits a local maximum at $\eta \sim 1.38$, exceeding the value predicted by (3.20), and even exceeding $w / w_{0}=1$. The difference, however, is reasonably small relative to the local minimum value of $w^{*}$ at $\eta=0$. Great care must therefore be taken in the interpretation of inferred axial velocity fields, since similar combinations of wake-like and jet-like flow have also been observed experimentally in developing wing-tip vortices using non-optical local measurement techniques, occurring likely as a consequence of the incomplete entrainment of secondary vortical structures formed around the tip (Birch et al. 2004).

The magnitude of $w^{*} / w_{0}$ at $\eta=0$ may also be approximated, and used as a quantitative measure of the error in the inferred axial velocity profile. If it is again assumed that $A \ll 1$, then $u^{\prime}$ can be obtained explicitly from (3.2), and is readily differentiable with respect to $\eta$. For $\eta \rightarrow 0$, the result yields (to a second-order approximation),

$$
\frac{\partial u^{\prime}}{\partial \eta}=\left(\alpha+\frac{1}{2}\right)^{2} \frac{v_{0}^{2}}{C r_{c}} .
$$




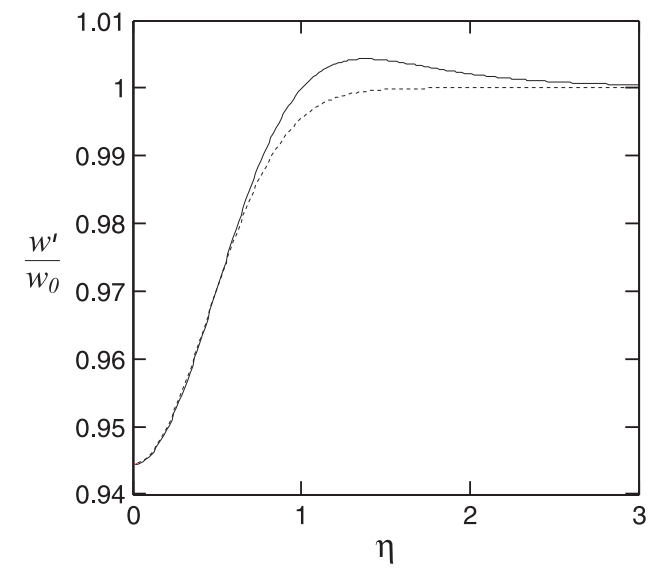

Figure 6. Typical profile of 'apparent' axial velocity for a vortex having $R e_{c}=6 \times 10^{5}$ and $C r_{c} / v_{0}=84.7$ (corresponding to oil particles $4 \mu \mathrm{m}$ in diameter).,$-(3.20) ;---,(2.2)$.

Also, for very small $\eta,(3.19)$ shows that $u^{\prime} / \eta$ is of $\mathcal{O}(\eta)$ and therefore vanishes. Furthermore, $v_{0}$ and $r_{c}$ can be expressed as functions of $z$ as

$$
\begin{aligned}
& v_{0}=\frac{\Gamma_{0}}{2 \pi r_{c}}\left(\frac{2 \alpha}{1+2 \alpha}\right) \\
& r_{c}=\sqrt{4 \alpha \nu \frac{z}{w_{0}}},
\end{aligned}
$$

where $w_{0}$ here is taken as the streamwise advection velocity scale and $\Gamma_{0}$ is the total circulation. Substituting (3.22) and (3.21) into (3.20) and integrating yields,

$$
w^{*}(0)=\left(\alpha+\frac{1}{2}\right)^{2} \frac{v_{0}^{2}}{C r_{c}} \frac{z-z_{0}}{r_{c}},
$$

where $z_{0}$ is a constant dependent upon the boundary conditions. Since $z$ remains an unknown function of the flow and particle parameters, (3.23) may be re-cast in the form

$$
\frac{w_{0}-w^{*}(0)}{v_{0}}=\frac{v_{0}}{C r_{c}} f\left(\frac{z}{r_{c}}\right),
$$

where the function $f$ has absorbed the constants. The function $f\left(z / r_{c}\right)$ appears to be fairly insensitive to changes in $v_{0}$ and $C$, and figure 7 shows the variation of $f$ with $r_{c} / r_{0}$ (where $r_{0}$ is an arbitrary fixed reference length scale for the axial profile, and $w_{0}=0$ ).

Equation (3.24) may again be compared with (3.12), yielding

$$
S_{k}=4 \frac{w_{0}-w^{*}(0)}{v_{0}}\left(\alpha+\frac{1}{2}\right) f\left(\frac{z}{r_{c}}\right)^{-1} .
$$

To achieve a $1 \%$ error in the inferred axial velocity profile, a Stokes number of $0.070 / f$ is required. consequently, the required particle size decreases very rapidly with decreasing vortex size.

\subsection{Effect of particle diameter distributions}

In the previous analyses, it has been assumed that the particles are all of equal diameter $d$. In practice, however, tracer particles will exhibit some distribution of diameters about a given mean. The standard deviation of the particle diameters, $\sigma$, will depend on the particle production technique (which, for the case of oil atomizers, may also be loosely 


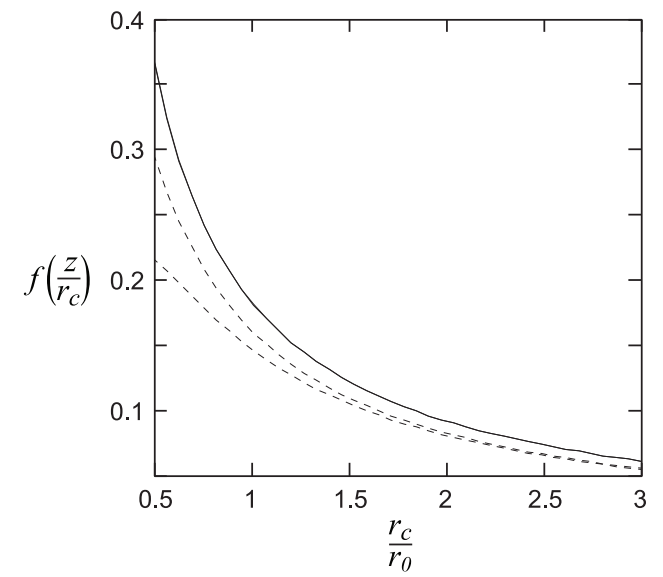

Figure 7. Variation of the function $f\left(z / r_{c}\right)$ with core radius for $C=3.6 \times 10^{3} \mathrm{~s}^{-1}$ and $3.6 \times 10^{5} \mathrm{~s}^{-1}$, and $\left.r_{0}=0.1 \mathrm{~m}\right) . \stackrel{\longrightarrow}{\longrightarrow}, v_{0}=20 \mathrm{~m} / \mathrm{s} ;---, v_{0}=10 \mathrm{~m} / \mathrm{s}$.

coupled to the particle production rate) and may be obtained from the captured particle images themselves (Martin et al. 1997). The magnitude of $A$, as a measure of the particle tracking error, should therefore be computed as an average over the distribution of particle diameters. Substituting (2.7) into (3.8),

$$
A=2\left(\alpha+\frac{1}{2}\right)^{2}\left(\frac{v_{0}}{r_{c}} \frac{\phi}{18 \nu}\right)^{2} d^{4} .
$$

If it is assumed that the particle diameters are normally distributed, the resultant mean tracking error amplitude $\bar{A}$ may be taken as the convolution of (3.26),

$$
\bar{A}=\frac{1}{\sqrt{2 \pi \sigma^{2}}} \int_{-\infty}^{\infty} 2\left(\alpha+\frac{1}{2}\right)^{2}\left(\frac{v_{0}}{r_{c}} \frac{\phi}{18 \nu}\right)^{2}(d-\xi)^{4} \exp \left(-\frac{\xi^{2}}{2 \sigma^{2}}\right) d \xi
$$

Because the velocity vectors obtained from PIV measurements are normally obtained by the simultaneous correlation of the particle images in the spectral domain, and because the correlations are carried out independently over small interrogation regions containing few particles, (3.27) may only be used as a limiting approximation. Integrating over the infinite domain yields a finite mean $\bar{A}$, which may be expressed in terms of $A$ as

$$
\bar{A}=1+6\left(\frac{\sigma}{d}\right)^{2}+3\left(\frac{\sigma}{d}\right)^{4}
$$

This relationship is also plotted in Figure 8. Significantly, (3.28) demonstrates that the value of $\bar{A}$ varies with $\sigma / d$, so that wider particle distributions will necessarily increase the tangential velocity tracking error. Given that, in many cases, $\sigma$ and $d$ are of similar order, and that $\bar{A} / A$ increases rapidly with increasing $\sigma / d$, the width of the particle distribution must also be considered alongside the mean particle diameter when assessing the suitability of PIV particles. Furthermore, because of the sensitivity of $\bar{A} / A$ to $\sigma / d$, even a small underestimation of the width of the distribution may have a significant effect upon the error. For example, Martinelli et al. (2007) report particle diameters in the range $d=1 \sim 2 \mu \mathrm{m}$; if this range of $\Delta d=1 \mu \mathrm{m}$ represents $6 \sigma$ (or $99.7 \%$ of all particles), the resultant $\bar{A}$ is $7.5 \%$ greater than $A$, but if the range reported represents $90 \%$ of all particles (a tolerance not uncommon for certain purpose-manufactured tracer particles), this increases to $27 \%$.

A similar convolution may be applied to (3.15) in order to assess the effect of a finite 


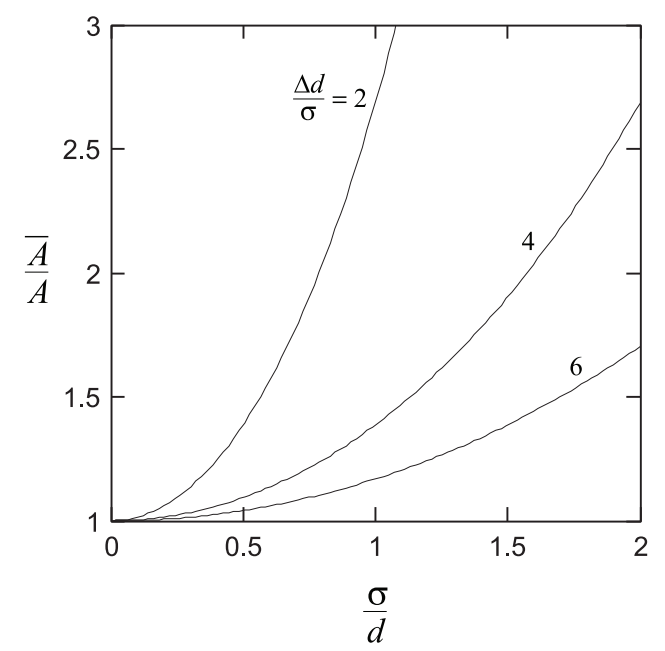

Figure 8. Variation of $\bar{A} / A$ with $\sigma / d$ for $\Delta d / \sigma=2,4$ and 6 .

particle distribution upon the peak measured radial velocity. The result yields,

$$
\frac{\overline{u_{0}^{\prime}}}{u_{0}^{\prime}}=1+\left(\frac{\sigma}{d}\right)^{2}
$$

where $\overline{u_{0}^{\prime}}$ is the mean peak particle radial velocity. Consequently, a finite distribution of particle diameters will also increase the particle radial velocities, but the effect of nonzero $\sigma$ upon $u_{0}^{\prime} / v_{0}$ is smaller than the effect upon $A$ by a factor of at least 6 .

\section{Vortices with arbitrary radial velocity profiles}

The analysis presented in $\S 3.1$ may equally be applied to a vortex having an arbitrary radial velocity distribution $u(\eta)$. Because vortices tend to evolve only very slowly in time (or downstream distance, for the case of trailing vortices), $u \ll v$; the measurements of Phillips \& Graham (1984) showed that $u$ remained typically two orders of magnitude smaller than $v$ even in the extreme cases of vortices with strong forced axial gradients. Equation (3.1) should therefore be a reasonable (if less accurate) approximation for the more general case of $u \neq 0$. To verify the validity of this assumption, a numerical solution was produced for the case of $u(\eta)=0.03 v(\eta)$ (a profile which is similar to those reported by Phillips \& Graham (1984), and satisfies continuity with (2.2) if $w_{0}(z)$ is approximated to first-order and $r_{0}=r_{c}$ ). Figure 9 compares $v / v^{\prime}-1$ to (3.1), and shows that the introduction of the nonzero radial velocity profile does not significantly affect $A$ or the general form of the radial distribution. Furthermore, for small $\eta$, the error between $v / v^{\prime}-1$ and (3.1) will become independent of $B$, increasing the accuracy of the error estimates.

By requiring that $u \neq 0$ and repeating the analysis described in $\S 3.1$, assuming that $A \ll 1$ and $d u / d t \ll v^{2} / r$, an approximation of $A$ can be obtained for $\eta \rightarrow 0$ as

$$
A=2\left(\alpha+\frac{1}{2}\right) \frac{v_{0}}{C r_{c}}\left(\psi+\left(\alpha+\frac{1}{2}\right) \frac{v_{0}}{C r_{c}}\right),
$$

where

$$
\psi=\lim _{\eta \rightarrow 0}\left(\frac{u(\eta)}{v(\eta)}\right)
$$




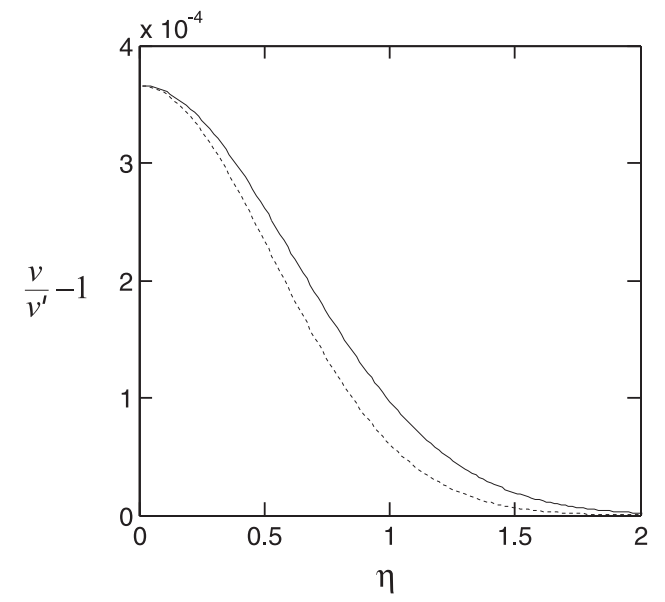

Figure 9. Particle tracking error in tangential velocity profile for the case $R e_{c}=6 \times 10^{5}$ and $C r_{c} / v_{0}=340$, with $u(\eta)=0.03 v(\eta) .-, v / v \prime-1 ;---,(3.1)$ with $B=1.80$.

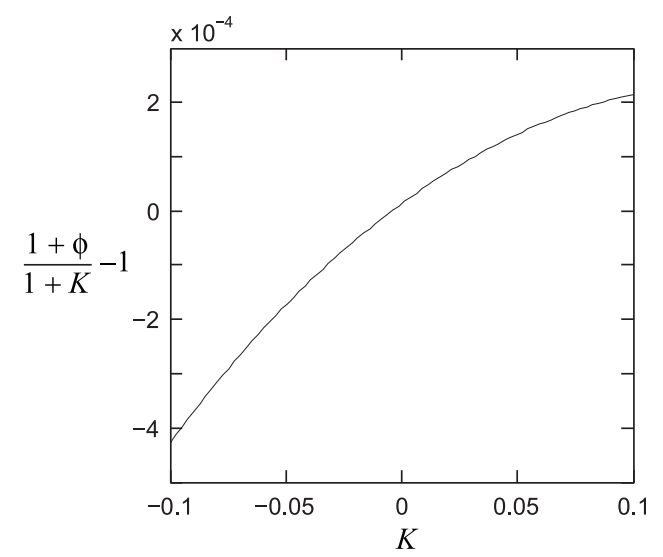

FiguRE 10. Variation between the value of $\psi$ obtained from the numerical solution of (4.1) and $K$, for the special case of $u=K v$.

and can be readily estimated from experimental measurements. For the limiting case of $\psi=0,(4.1)$ becomes identical to (3.8). This expression also demonstrates that the effect of the radial velocity upon the tangential velocity tracking error will dominate for cases where $\psi \gg v_{0} / \mathrm{Cr}_{c}$, so this criterion may be used to assess the validity of the assumption of small $u$ in $\S 3.1$ and $\S 3.2$.

Because (3.1) cannot be shown to be valid for arbitrary $u(\eta)$, and because for arbitrary $u(\eta)$ the maximum error does not necessarily occur at $\eta=0,(4.1)$ may only be used as an approximation. To further examine the validity of the assumption, a more general simplified case of $u(\beta \eta) / v(\eta)=K$ was considered, where $\beta$ is a radial scaling factor. These profiles, again, are not too dissimilar those presented by Phillips \& Graham (1984), and satisfy continuity with (2.2). The magnitude of the tangential velocity tracking error for cases with $-0.1 \leq K \leq 0.1$ and $0.8 \leq \beta \leq 1.2$ was determined from the numerical solution, and the values of $\psi$ satisfying (4.1) were determined and compared to $K$. For all of the test cases with $\beta=1,(4.1)$ was accurate to within $0.05 \%$ (Figure 10). For all of the more general cases tested with $\beta \neq 1$, the difference between the imposed value of $K$ and the estimated value $\psi$ remained less than $3 \%$. 


\begin{tabular}{lccccccc} 
Fluid & $\phi$ & $d(\mu \mathrm{m})$ & $\sigma / d$ & $\bar{A} / A$ & $C\left(\mathrm{~s}^{-1}\right)$ & $C r_{c} / v_{0}$ & $R e_{c}$ \\
\hline Water & 1.65 & 14 & $\sim 0.31$ & $\sim 1.604$ & $5.6 \times 10^{4}$ & $4.39 \times 10^{4}$ & 13.8 \\
Air & 650 & 1.5 & $\sim 0.11$ & $\sim 1.073$ & $2.1 \times 10^{5}$ & $3.11 \times 10^{3}$ & 1590
\end{tabular}

TABLE 1. Key particle and vortex scaling parameters. Note that the particle diameters and standard deviations listed above are taken from the original manufacturer specifications, assuming normal distributions.

\section{Experimental demonstration}

The analysis presented above necessarily requires the assumption that $u=0$, or the less accurate but reasonable approximations of $u \ll v$ and $d / d z \ll d / d r$. In practice, however, the production of entirely axisymmetric vortices with an absence of axial gradients can be extremely difficult; the vortices produced by finite wings and vortex generators can exhibit strong axial gradients (see, for example, Phillips \& Graham 1984). The method of Meunier et al. (2002) has therefore been adopted here, whereby an impulsively-started, high-aspect-ratio lifting plate is used to produce a nominally two-dimensional, stationary, axisymmetric Batchelor vortex.

\subsection{Experimental apparatus}

Experiments were carried out in both quiescent air and water, inside a specially constructed, sealed tank (having internal dimensions $0.64 \mathrm{~m} \times 0.64 \mathrm{~m} \times 0.2 \mathrm{~m}$ ) in order to minimize background motion. A thin, sharp-edged rectangular plate with chamfered corners (having a chord of $c=0.2 \mathrm{~m}$ and spanning the depth of the tank) was impulsively caused to translate and rotate through the fluid by means of a computer-controlled, 4 degree-of-freedom servo-traverse. The motion profile of the lifting plate was carefully tuned to produce a stable laminar vortex which was as strong and axisymmetric as possible. The vortex axis was vertical, in order to minimize the influence of gravity or buoyancy on the tangential and radial components of the particle velocities. The background motion within the tank was allowed to decay naturally between experimental runs, and the standard deviation of background vorticity levels was typically $0.1 \% \sim$ $0.2 \%$ of the peak experimental values.

The tracer particles used were commercially available, large-diameter silver-coated hollow glass spheres (TSI model 10089-SLVR) and oil droplets produced by an atomizer (TSI model 9307) for the measurements in water and air, respectively. The physical properties of the tracer particles are listed in table 1, together with the corresponding vortex Reynolds numbers $R e_{c}=2 \pi r_{c} v_{o} / \nu$.

Instantaneous cross-flow velocity fields at the centre of the tank were obtained using a particle-image velocimetry system with a maximum sampling frequency of $10 \mathrm{~Hz}$. The tracer particles were illuminated using dual $50 \mathrm{~mJ}$ Nd:YAG lasers expanded into light sheet of thickness $\sim 1 \mathrm{~mm}$ using a cylindrical lens. The lasers were computer-triggered, and were synchronized with the lifting plate motion-control system. Image pairs were collected using a CCD camera system having $2048 \times 2048$ pixels, with optical magnification yielding spatial resolutions of $6.04 \mu \mathrm{m} /$ pixel and $117 \mu \mathrm{m} /$ pixel for measurements in air and water, respectively, in order to achieve particle images of at most 2 pixels in diameter. Image-pair time-delays of $7.5 \times 10^{-4} \mathrm{~s}$ in air and $4 \times 10^{-3} \mathrm{~s}$ in water were used. The direct-correlation method of Hart (2000) was applied, using a typical interrogation windows size of $48 \times 48$ pixels with no overlap, though smaller interrogation windows 


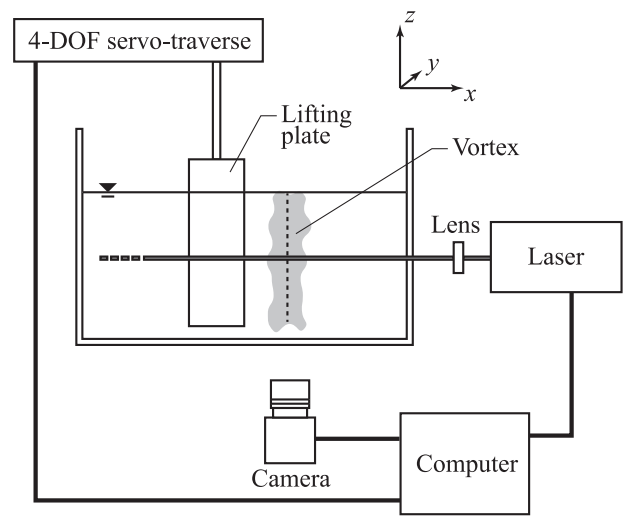

FiguRE 11. Schematic of experimental setup (not to scale).

were used to verify measurements in regions with high velocity gradients. A centraldifferencing offset was used in order to achieve higher-order accuracy (see, for example Wereley \& Meinhart 2001). Sub-pixel interpolation of the cross-correlation peaks was carried out using a 6-point Gaussian fit. Figure 11 shows a schematic of the experimental and optical apparatus.

The velocity fields were conditioned only by the substitution of bad data points (generally resulting from poor signal-to-noise ratios in the correlation plane) with interpolated values; typically no more than $0.2 \%$ of vectors were identified as being artefacts of the PIV analysis. The velocity fields were also corrected for any bulk translation or 'wandering' of the vortex by subtracting the velocity at the vortex centre from the entire field. No other corrections have been implemented.

The axial vorticity field $\zeta_{z}(x, y)$ was obtained by second-order numerical differentiation, and the centre of the vortex was identified as the location of maximum vorticity with sub-grid interpolation. Planes parallel to the vortex axis were also sampled in order to ensure that axial velocity components were small and limited to zero-mean noise. The spanwise vorticity levels $\zeta_{x}$ were used as an indication of the axial gradients, as this quantity is insensitive to misalignment between the vortex axis and the PIV image axes. A typical field of $\zeta_{x} / \zeta_{z 0}$ (where $\zeta_{z 0}$ is the axial vorticity at the vortex centre) in a plane parallel to the vortex axis is shown in figure 12 . In the vicinity of the vortex, the spanwise vorticity remains less than $\sim 1 \%$ of the peak axial vorticity.

The error in the PIV measurements of the instantaneous cross-flow velocity profiles has been estimated to be typically $1 \%$ based on conventional error estimation techniques (see Tropea et al. 2007). The error in the tangential velocity fields is larger $(\sim 2 \%)$, owing to the additional uncertainty in determining the location of the vortex centre. For the more critical instantaneous radial velocity profiles, however, local experimental uncertainty may have been as high as $40 \%$ owing primarily to the finite resolution of the PIV system used. Because of this level of uncertainty, the experimental results which follow must be interpreted with caution.

\subsection{Results}

Figure 13 shows contours of normalized axial vorticity and tangential velocity from single PIV realizations of vortices produced in air and water. The vorticity fields exhibit a good degree of axisymmetry within the core region, though the asymmetry introduced by the 


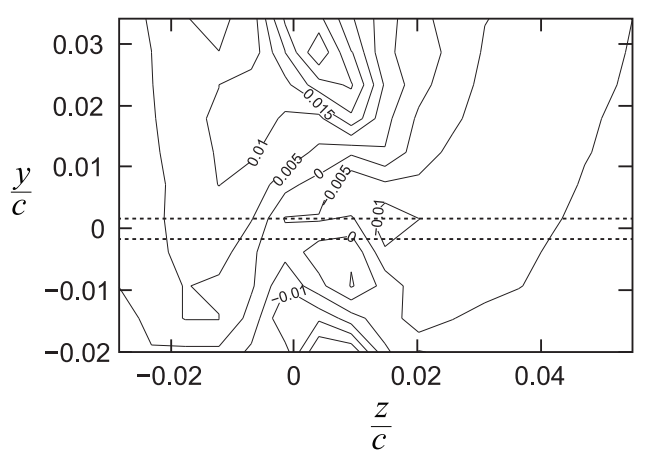

Figure 12. Typical contours of $\zeta_{x} / \zeta_{z 0}$ in air. The origin corresponds approximately to the intersection of the vortex axis and the centre of the tank (where cross-flow velocity fields were obtained). - -,$- y= \pm r_{c}$.

ongoing roll-up of the lifting plate shear layer becomes more significant for $\eta \gtrsim 1$. The tangential velocity fields are less symmetric, as these are also highly sensitive to error in the location of the vortex centre and bulk-motion velocity.

In order to obtain profiles of $v(\eta)$ and $u(\eta)$, circumferential averages of these velocity components were computed over the sector $\pi \leq \theta \leq 2 \pi$, as this region of the velocity field exhibited the highest degree of axisymmetry, and was least affected by the lifting plate wake. Since $C \gg v_{0} / r_{c}$, the characteristic timescale of the tracer particles is much smaller than the circumferential convection time, and the restriction of circumferential averages to a sector of the field is not expected to affect the applicability of the assumptions made in $\S 3$. The centre of the vortex was identified as the location of maximum local vorticity, and $r_{c}$ was taken as the radius at which the circumferentially-averaged tangential velocity achieved a local maximum. Figure 14 shows the circumferentially-averaged profiles of $v(\eta)$. In both air and water, the profiles agree very well with (2.1) for $0 \leq \eta \lesssim 1.5$; this is as expected, since $A$ is of $\mathcal{O}\left(10^{-7}\right)$. For larger $\eta$ (not shown), the agreement breaks down as a result of the influence of the lifting plate shear layer.

Of more interest, perhaps, is the radial velocity profile. Because of the typically small magnitudes of $u$, however, the identification of the location of the vortex centre and bulk translation velocity present another major source of error. Both of these will have the effect of imposing a symmetric distribution of noise amplitude, but will be minimized by spatial averaging. The effect of these uncertainties upon the mean profiles obtained using the current conditional spatial averaging was investigated by applying the transformation to a synthetic velocity field described by a two-dimensional Batchelor vortex with $u=0$, with imposed errors. For small errors in the location of the vortex centre $\left(\sim 1 \%\right.$ of $\left.r_{c}\right)$, $u(\eta)$ develops a strong local peak at $\eta=0$, while for small bulk translational velocities $\left(\sim 1 \%\right.$ of $\left.v_{0}\right), u(\eta)$ is nonzero at $\eta=0$ and increases in magnitude with increasing $\eta$.

Figure 15 shows the normalized profiles of $u(\eta)$ from the vortices produced in air and water, which agree surprisingly well (for $\eta \lesssim 1.5$ in air and $\eta \lesssim 1$ in water) with (3.17). The profiles both show $u \sim 0$ for $\eta \sim 0$ and exhibit a local maximum at around $\eta \sim 0.6$, which together suggest that the contamination of the results by the sources of error discussed above is minimal. A small linear shift in $\eta$ (typically $\sim 0.05$ ) was observed in the experimental results; this was attributed to the asymmetry of the vortex and was corrected.

The magnitudes of the peak radial velocity were, however, significantly underpredicted by (3.15), even when these are corrected for the distribution of particle diameters using (3.29). While it is possible that the good agreement between the experimental results 

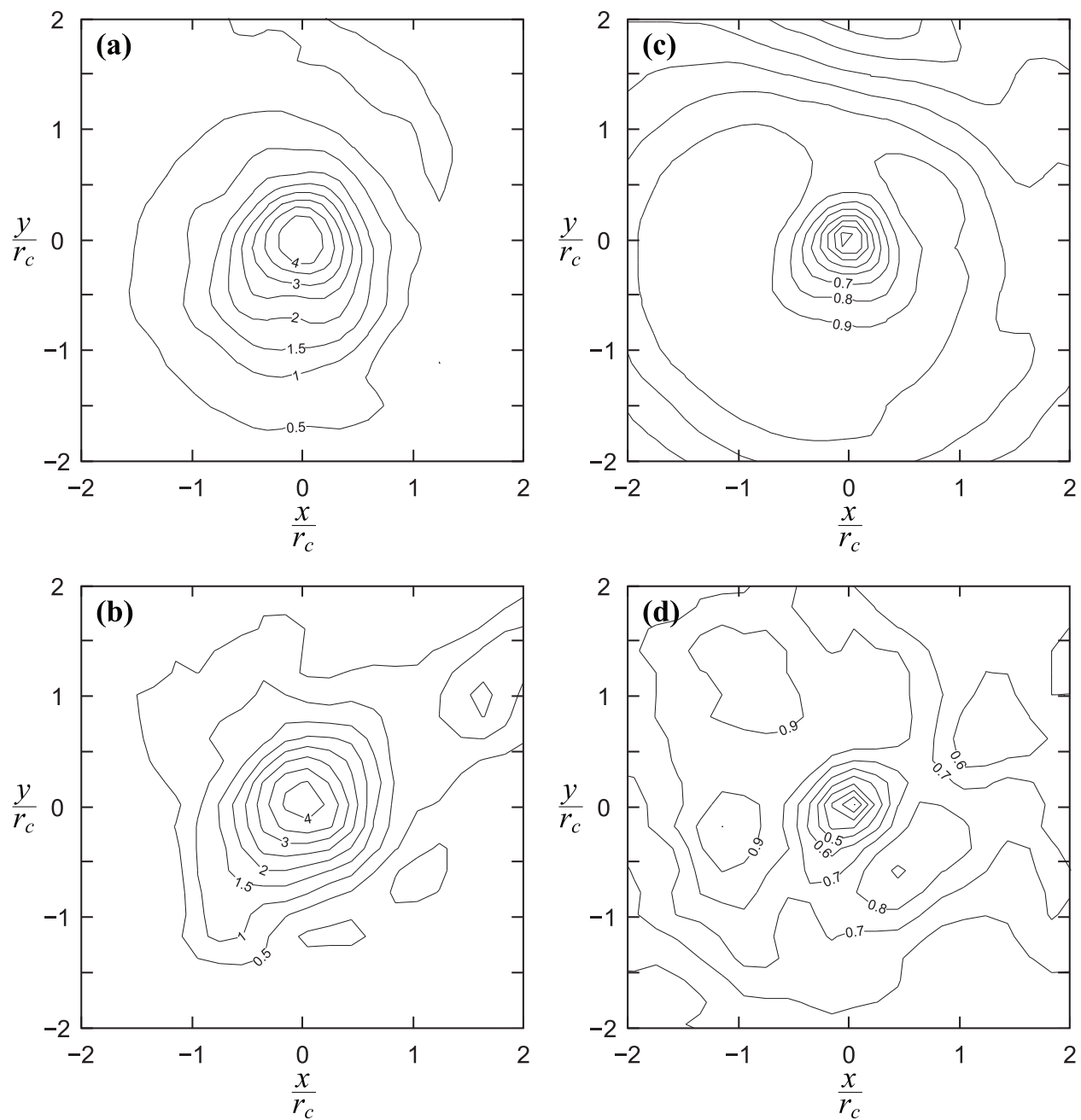

Figure 13. Contours of $\zeta_{z} r_{c} / v_{0}$ in (a), air and (b), water; contours of $v / v_{0}$ in (c), air and (d), water.

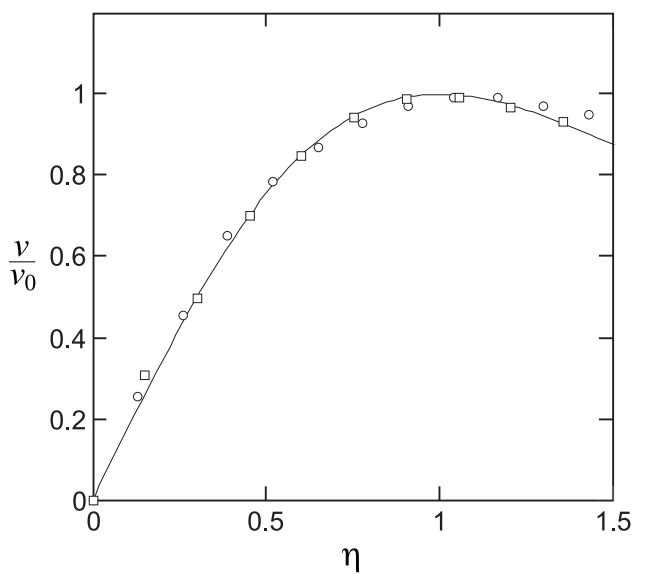

FIgURE 14. Conditional circumferential average of tangential velocity component. ॰, air; $\square$, water; $\longrightarrow,(2.1)$. 


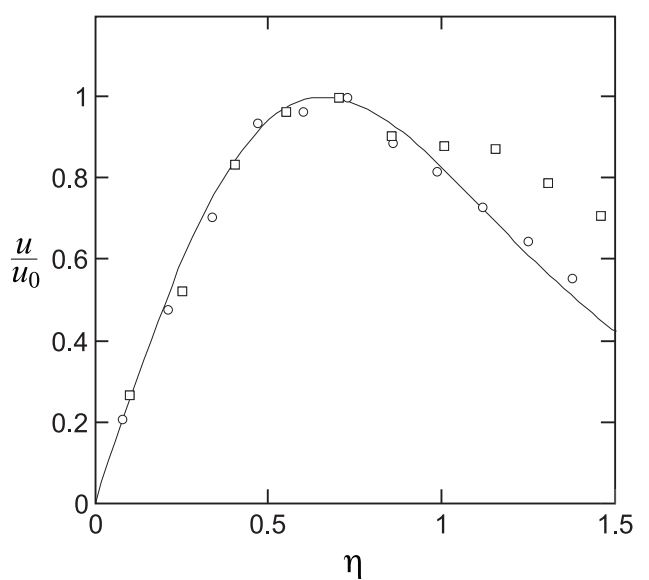

FIGURE 15. Self-scaled conditional circumferential average of radial velocity component. ○, air; $\square$, water; $-(3.17)$.

and (3.17) was entirely spurious, the analysis in $§ 3.4$ suggests that the predicted particle velocity profiles will be highly sensitive to uncertainties in experimental parameters, and that tracking error estimates will tend to be nonlinearly amplified by increasing uncertainty. Consequently, the uncertainty in the radial velocity scale $v_{0}^{2} / C r_{c}$ was considered in closer detail.

While the error in determining $v_{0}$ and $r_{c}$ from a given experimental data set is important, these quantities can be obtained from direct measurements and the effect of their respective uncertainties upon the profiles has already been discussed. It is therefore expected that the uncertainty in the magnitude will be dominated by $C$. Since the only quantity in $C$ which cannot be directly measured or inferred is the particle diameter, and since $C \propto 1 / d^{2}$, the uncertainty in $d$ will dominate the error band of $C$.

For the case of the oil droplets in air, light scattering effects precluded the direct characterization of particles from the PIV images. However, owing to the method of particle generation and the mean particle advection time during a typical experiment, there is a reasonable expecta

The solid tracer particles used in water, on the other hand, could be easily characterized; the silver coating facilitated direct imaging by scanning-electron microscopy. Figure 16 (a) shows a composite image of a small random sample of typical particles, together with the microscope scale markings. The images reveal that the particles are highly spherical. However, even in this very limited sample size, there is a wide distribution of diameters (particles with diameters ranging from $\sim 3 \mu \mathrm{m}$ to $\sim 25 \mu \mathrm{m}$ are apparent in this image), and this distribution is neither symmetric nor Gaussian. Furthermore, there is evidence of the presence of particles of foreign matter within the sample which are large relative to the mean diameter of the PIV particles themselves (figure $16 \mathrm{~b}$ ). There is also evidence of particle agglomeration around the foreign matter, though it is impossible to determine from these images the cause of the agglomeration or whether these particles would separate once immersed in water. Though inconclusive, the evidence in the micrographs is consistent with the underprediction of the radial velocity scale by (3.29).

Finally, the inferred axial velocity profiles were obtained by assuming axisymmetry and applying the continuity equation, taking $w_{0}=0$. Figure 17 shows the approximated profiles of $w(\eta)$. Though the uncertainty in this particular quantity is necessarily large, the profile shows a reasonably good agreement with (2.2), as predicted by (3.20), though 

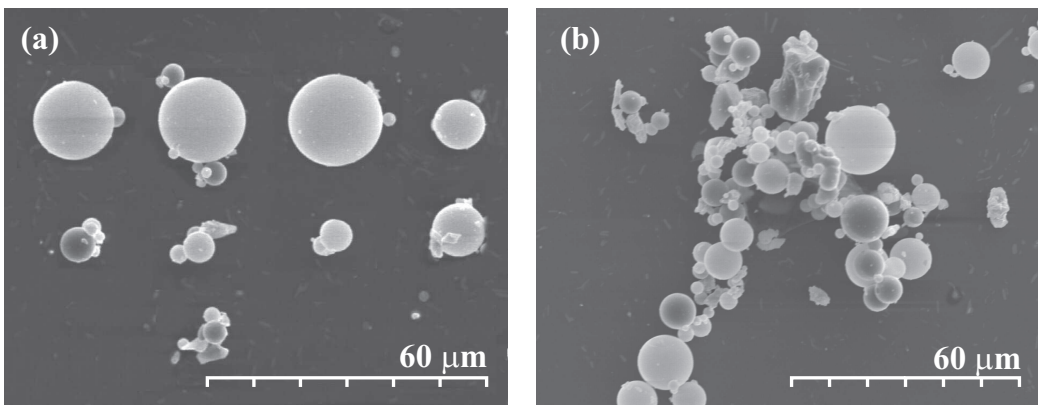

FIGURE 16. (a), Composite scanning-electron micrograph image of a random selection of tracer particles used in water. (b), Example of a concentration of foreign matter and surrounding particle agglomeration.

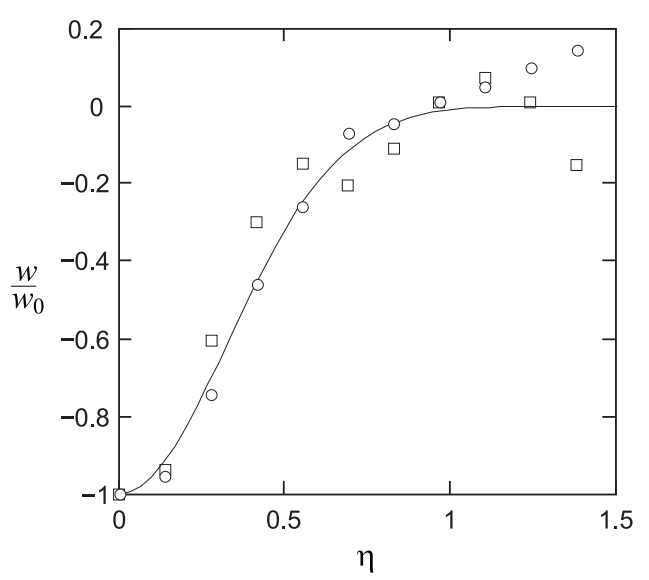

FiguRE 17. Self-scaled inferred axial velocity component. o, air; $\square$, water;

$(2.2)$

the small overshoot apparent in the numerical solutions could not be identified owing to the uncertainty. As with the radial velocity profiles, the agreement is better in air than in water.

\section{Discussion}

The approximate model for the transport of tracer particles within a Batchelor vortex yielded estimates of the error between the particle and fluid velocities in all three spatial dimensions. This error, at least within the Batchelor vortex, was highly anisotropic; the common practice of using $S_{k}<0.1$ as a tracer particle selection criterion may not yield optimal results for measurements within strong vortex flows. To achieve a given error margin on $u$ or $v$ (or even $w$, as inferred from continuity), the maximum limit on $S_{k}$ can vary by as much as an order of magnitude and is strongly dependent upon the distribution of particle diameters.

In all cases, the error scales with the dimensionless quantity $v_{0} / C r_{c}$, or the ratio of characteristic time-scales between the particle and the vortex, which agrees with the more generalized models of Maxey \& Riley (1983) and Wang \& Stock (1993). The error in the tangential velocity profile in particular is shown to be proportional to $\left(v_{0} / C r_{c}\right)^{2}$, demonstrating that the tangential velocity is far less susceptible to particle momentum effects than the radial (or inferred axial) velocity profiles. For the case of PIV measurements 
within Batchelor vortices, then, field or integral quantities strongly dependent upon $v$ and $\partial v / \partial r$ (such as the axial vorticity and circulation) may be obtained from experimentally measured velocity fields with a reasonable degree of confidence using particles substantially larger than those normally recommended. On the other hand, the selection of particles using the conventional Stokes number criterion will result in a larger amount of error in the normalized radial velocity profile than expected.

The experimental demonstration of these analytical results was complicated by the difficulty in producing a stationary, two-dimensional, axisymmetric Batchelor vortex in quiescent fluid, as well as by the very high level of experimental uncertainty in measurements of $u$ using standard two-frame PIV techniques. Nevertheless, experimental results were obtained for two different cases of seeded vortex flows having an order of magnitude difference in $v_{0} / C r_{c}$ and two orders of magnitude difference in Reynolds number. In both cases, there was remarkably good agreement between the measured and predicted selfscaled tangential and radial velocity profiles, but large differences in the radial velocity scaling. Together with the high degree of experimental uncertainty, this may indicate that the good agreement of the normalized profiles was entirely spurious, and the experimental results must therefore be interpreted with care. However, the radial velocity magnitude was shown to be very sensitive to the mean particle diameter, the distribution of particle diameters may have been highly non-normal, and the particles may have undergone some agglomeration. Though no conclusive evidence is available, these effects could have been the cause of the poor agreement in the radial velocity scaling.

\section{Conclusions}

An approximate solution for the classical model of the transport of small spherical particles in two-dimensional $(u=0)$ and three-dimensional $(u \neq 0)$ Batchelor $q$-vortices has been presented. To within a reasonable approximation, the tracking error in the tangential velocity is found to vary universally with $\eta$ for cases of $u=0$. For cases with $u \neq 0$ but $u \ll v$, the approximate solution remains reasonable. The peak error in the tangential velocity is found to vary with the square of the nondimensional parameter $v_{0} / \mathrm{Cr}_{c}$, though this error may increase significantly if the particle diameters are normally distributed about a mean value. For vortices with $u=0$, a nonzero radial velocity profile will be apparent in PIV measurements, with a peak radial velocity occurring at $\eta \sim 0.66$, independent of the flow and particle properties. The nonzero radial component will give rise to an inferred axial velocity profile in order to satisfy continuity, and this inferred axial velocity profile will tend to agree well with the form of $w$ predicted by the Batchelor solution. An approximation of the maximum particle Strouhal numbers such that the particle tracking error in each of $u, v$ and $w$ remains below $1 \%$ has also been presented. An experimental demonstration of this model was also carried out, though the results are subject to large uncertainty. Reasonable agreement between the self-normalized radial velocity profiles and the model is demonstrated, but the velocity scaling was significantly underpredicted as a consequence of the uncertainty. Conclusive validation is therefore impossible, but the results are consistent.

\section{Acknowledgements}

This work was supported by the U.K. Engineering and Physical Sciences Research Council under grant number EP/H030360/1. DMB is grateful to Professor Alan G. Robins for very helpful discussions and advice. 


\section{REFERENCES}

Bailey, S. C. C. \& Tavoularis, S. 2008 Measurements of the velocity field of a wing-tip vortex, wandering in grid turbulence. J. Fluid Mech. 601, 281-315.

Bailey, S. C. C., Tavoularis, S. \& Lee, B. H. K. 2006 Effects of freestream turbulence on wing-tip vortex formation and near field. J. Aircraft 43 (5), 1282-1291.

Bandyopadhyay, P., Stead, D. \& Ash, R. 1991 Organized nature of a turbulent trailing vortex. AIAA J. 29, 1627-1633.

Batchelor, G. K. 1964 Axial flow in trailing line vortices. J. Fluid Mech. 20, 645-658.

Beninati, M. \& Marshall, J. 2005 An experimental study of the effect of free-stream turbulence on a trailing vortex. Exp. Fluids 38, 244-257.

Beresh, S. J., Henfling, J. F. \& Spillers, R. W. 2010 Meander of a fin trailing vortex and the origin of its turbulence. Exp. Fluids 49, 599-611.

Birch, D. M. 2012 Self-similarity of trailing vortices. Phys. Fluids 24 (3), 1-16.

Birch, D. M. \& LEE, T. 2005 Investigation of the near-field tip vortex behind an oscillating wing. J. Fluid Mech. 544, 201-241.

Birch, D. M., Lee, T., Mokhtarian, F. \& Kafyeke, F. 2004 Structure and induced drag of a tip vortex. J. Aircraft 41 (5), 1138-1145.

Brown, M. R., Macinnes, J. M. \& Allen, R. W. K. 2007 Three-component micro-piv using the continuity equation and a comparison of the performance with that of stereoscopic measurements. Exp. Fluids 42 (2), 197-205.

Chow, J., Zilliac, G. \& Bradshaw, P. 1997 Mean and turbulence measurements in the near field of a wingtip vortex. AIAA J. 35 (10), 1561-1567.

Dacles-Mariani, J., Zilliac, G. G., Chow, J. S. \& Bradshaw, P. 1995 Numerical/experimental study of a wingtip vortex in the near field. AIAA J. 33 (9), 1561-1568.

Devenport, W. J., Rife, M. C., Liapis, S. I. \& Follin, G. J. 1996 The structure and development of a wing-tip vortex. J. Fluid Mech. 312, 67-106.

Dring, R. P. 1982 Sizing criteria for laser anemometry particles. J. Fluids Eng. 104 (1).

Goto, S. 2008 A physical mechanism of the energy cascade in homogeneous isotropic turbulence. J. Fluid Mech. 605, 355-366.

Grant, I., Pan, X., Wang, X. \& Stewart, N. 1994 Correction for viewing angle applied to piv data obtained in aerodynamic blade vortex interaction studies. Exp. Fluids 18 (1-2), 95-99.

Greenwell, D. I. 2002 Effect of tracer particle characteristics on visualization of delta wing vortices. Aeronautical J. 106 (1063), 473-482.

HART, D. P. 2000 Super-resolution PIV by recursive local-correlation. J. Visualization 3 (2), 187-194.

Hoffman, E. R. \& Joubert, P. N. 1963 Turbulent line vortices. J. Fluid Mech. 16, 395-411.

Iungo, G. V., Skinner, P. \& Buresti, G. 2009 Correction of wandering smoothing effects on static measurements of a wing-tip vortex. Exp. Fluids 46, 435-452.

Kriebel, A. R. 1961 Particle trajectories in a gas centrifuge. Journal of Basic Engineering 83 (3), 333-340.

Leishman, J. G. 1996 Seed particle dynamics in tip vortex flows. J. Aircraft 33 (4), 823-825.

Martin, W. T., Kadambi, J. R. \& Wernet, M. P. 1997 Particle imaging technique for size, velocity, and concentration measurements in particle laden flows. Proc. SPIE - Int. Soc. Optical Eng. 3172, 518-529.

Martinelli, F., Olivani, A. \& Coghe, A. 2007 Experimental analysis of the precessing vortex core in a free swirling jet. Exp. Fluids 42, 827-839.

Maxey, R. \& Riley, J. J. 1983 Equation of motion for a small rigid sphere in a nonuniform flow. Phys. Fluids 26 (4), 883-889.

Melling, A. 1997 Tracer particles and seeding for particle image velocimetry. Meas. Sci. Technol. 8, 1406-1416.

Meunier, P., Ehrenstein, U., Leweke, T. \& Rossi, M. 2002 A merging criterion for twodimensional co-rotating vortices. Phys. Fluids 14 (8), 2757-2766.

Paiva, J., Salcedo, R. \& Araujo, P. 2010 Impact of particle agglomeration in cyclones. Chem. Eng. J. 162 (3), 861-876. 
Phillips, W. R. C. 1981 The turbulent trailing vortex during roll-up. J. Fluid Mech. 105, 451-467.

Phillips, W. R. C. \& Graham, J. 1984 Reynolds-stress measurements in a turbulent trailing vortex. J. Fluid Mech. 147, 353-371.

Ramasamy, M., Johnson, B. \& Leishman, J. G. 2009 Turbulent tip vortex measurements using dual-plane stereoscopic particle image velocimetry. AIAA J. 47 (8), 1826-1840.

Regunath, G. S., Zimmerman, W. B., Tesar, V. \& Hewakandamby, B. N. 2008 Experimental investigation of helicity in turbulent swirling jet using dual-plane dye laser piv technique. Exp. Fluids $\mathbf{4 5}$ (6), 973-986.

Rossow, V. J. 1999 Lift-generated vortex wakes of subsonic transport aircraft. Prog. Aerospace Sci. 35, 507-660.

SAffman, P. G. 1965 The lift on a small sphere in a slow shear flow. J. Fluid Mech. 22 (2), 385-400.

Spalart, P. 1998 a Airplane trailing vortices. Ann. Rev. Fluid Mech. 30, 107-138.

SPAlART, P. $1998 b$ On the far wake and induced drag of aircraft. J. Fluid Mech. 603, 413-430.

TARi, P. Hashemi, Gurka, R. \& Hangan, H. 2010 Experimental investigation of tornado-like vortex dynamics with swirl ratio: the mean and turbulent flow fields. J. Wind Eng. Ind. Aerodyn. 98, 936-944.

Tropea, C., Yarin, A.L. \& Foss, J.F. 2007 Springer Handbook of Experimental Fluid Mechanics. Springer.

Uzun, A., Hussaini, M. Y. \& Streett, C. L. 2006 Large-eddy simulation of a wing tip vortex on overset grids. AIAA J. 44 (6), 1229-1242.

van Jaarsveld, J. P. J., Holten, A. P. C., Elsenaar, A., Trieling, R. R. \& van Heijst, G. J. F. 2011 An experimental study of the effect of external turbulence on the decay of a single vortex and a vortex pair. J. Fluid Mech. 670, 214-239.

Wang, L.-P. \& Stock, D. E. 1993 Dispersion of heavy particles by turbulent motion. J. Atmos. Sci. 50 (13), 1897-1913.

Wereley, S. T. \& Meinhart, C. D. 2001 Second-order accurate particle image velocimetry. Exp. Fluids 31, 258-268.

Widnall, S. 1975 The structure and dynamics of vortex filaments. Ann. Rev. Fluid Mech. 7, $141-165$.

ZhANG, W. \& SARKAR, P. P. 2012 near-ground tornado-like vortex structure resolved by particle image velocimetry. Exp. Fluids 52, 479-493. 